\title{
Du centenaire de la Révolution Française à la Première Guerre mondiale
}

Une période féconde pour l'histoire moderne et contemporaine (les sociétés de l'Histoire de la Révolution française, d'Histoire moderne, d'Histoire de la Révolution de 1848)

From the Centenary of the French Revolution to the First World War: A productive period for Modern and Contemporary history

\section{(2) OpenEdition}

\section{Journals}

\section{Édition électronique}

URL : https://journals.openedition.org/ahrf/11117

DOI : 10.4000/ahrf.11117

ISSN : 1952-403X

Éditeur :

Armand Colin, Société des études robespierristes

Édition imprimée

Date de publication : 1 septembre 2008

Pagination : 45-62

ISBN : 978-2-200-92515-4

ISSN : 0003-4436

\section{Référence électronique}

Jacqueline Lalouette, "Du centenaire de la Révolution Française à la Première Guerre mondiale », Annales historiques de la Révolution française [En ligne], 353 I juillet-septembre 2008, mis en ligne le 01 septembre 2011, consulté le 01 juillet 2021. URL : http://journals.openedition.org/ahrf/11117 ; DOI : https://doi.org/10.4000/ahrf.11117

Ce document a été généré automatiquement le 1 juillet 2021.

Tous droits réservés 


\section{Du centenaire de la Révolution Française à la Première Guerre mondiale}

Une période féconde pour l'histoire moderne et contemporaine

(les sociétés de l'Histoire de la Révolution française, d'Histoire moderne, d'Histoire de la Révolution de 1848)

From the Centenary of the French Revolution to the First World War: A

productive period for Modern and Contemporary history

Jacqueline Lalouette

Lorsque la Société des études robespierristes fut créée, en 1907, d'autres sociétés historiques existaient depuis un temps plus ou moins long. Il faut signaler en premier lieu la Société d'histoire de France, fondée en 1833, reconnue d'utilité publique en 1851 et forte de 550 membres aux alentours de 1900. Contemporaine de la précédente, la Société des études historiques fut reconnue d'utilité publique plus tardivement, en 1872 ; elle rassemblait 255 membres à la fin du XIXe siècle. Suivirent la Société d'histoire du protestantisme français et la Société des études juives, créées respectivement en 1852 et 1882, et reconnues d'utilité publique, la première en 1870, la seconde en $1896^{1}$; entre-temps, la Société de l'histoire de l'art français avait vu le jour en 1872. Furent ensuite créées la Société de l'histoire de la Révolution française (1888), la Société d'histoire contemporaine (1890), la Société d'histoire moderne ${ }^{2}$ (1901), la Société d'histoire de la Révolution de 1848 (1904). Enfin, dans les années qui suivirent la fondation de la Société des études robespierristes, apparurent successivement la Société d'histoire du droit (1913) et la Société d'histoire ecclésiastique de la France (1914). Ainsi, en quatre-vingts ans environ, avec un pic correspondant à la période allant du centenaire de la Révolution française à la Première Guerre mondiale, se forma un ensemble imposant de sociétés historiques, dont les champs de recherche différaient, dont les orientations idéologiques pouvaient varier considérablement, mais qui étaient unies par un but commun: "Toutes ces sociétés ont pris l'initiative de recherches et de publications qui ont rendu de signalés services; elles ont contribué à 
rapprocher les historiens adonnés à des études voisines et stimulé leur énergie ; enfin, il en est, comme la Société d'histoire moderne, qui, en inscrivant au programme de leurs séances d'importantes questions de méthode et de critique, ont su prendre un ascendant marqué sur l'orientation même de tout le travail historique ${ }^{3}$ ».

C'est à la Société de l'histoire de la Révolution française [SHRF], à la Société d'histoire moderne [SHM] et à la Société d'histoire de la Révolution de 1848 [SHR de 1848] que nous nous intéresserons ici, présentant leurs fondateurs, le contexte dans lequel elles apparurent et les liens unissant leurs membres.

\section{La Société de l'histoire de la Révolution française}

L'origine de cette société remonte à 1881 , date à laquelle fut fondée la revue $L a$ Révolution française, à l'initiative d'Auguste Dide, de Jean-Claude Colfavru et d'Étienne Charavay; ces trois hommes, sous le patronage d'Henri Martin, d'Hippolyte Carnot, d'Édouard Charton et d'Eugène Pelletan, avaient voulu donner «une impulsion nouvelle aux études en les concentrant sur notre grande tradition nationale $»^{4}$. Les appels de La Révolution française, qui poussait à la création de sociétés locales pour la célébration du premier centenaire de la Révolution, trouvèrent un grand écho. En 1886, fut ainsi fondé le "Comité d'études pour la préparation historique du Centenaire de 1789 », que présida Hippolyte Carnot. Le 8 mars 1888, ce Comité se constitua en Société de l'histoire de la Révolution française, Hippolyte Carnot en étant président, Alphonse Aulard, secrétaire général et Étienne Charavay, secrétaire général adjoint et trésorier. D'après les statuts, l'objet de la Société était « de faire prévaloir la méthode scientifique dans les études sur la Révolution française, d'offrir un point de ralliement aux personnes qui, à Paris et dans les départements s'occupent de l'histoire de France depuis 1789 ; de publier des textes inédits ou rares et des œuvres originales touchant l'histoire de France depuis 1789, d'organiser des conférences historiques à Paris et dans les départements $»^{5}$. La Société était administrée par un Comité directeur de trentedeux membres, renouvelé par quart tous les ans, par un Bureau, composé d'un président, de trois vice-présidents, d'un secrétaire général et d'un secrétaire adjoint. Le 15 mars, Hippolyte Carnot mourut, et la Société demanda à son fils, Sadi Carnot, président de la République depuis le 3 décembre 1887, de lui succéder, ce qu'il accepta, à titre honorifique ${ }^{6}$. Édouard Charton fut porté à la présidence; décédé le 27 février 1890, il fut remplacé par Jules Claretie ${ }^{7}$, primitivement vice-président, aux côtés du sénateur Auguste Dide et du député Jean-Claude Colfavru. À cette date, le Comité directeur était le même qu'en 1888, à quelques exceptions près. Des parlementaires y siégeaient, le sénateur Jean Macé et quelques députés: Auguste Burdeau, Antonin Dubost, Anatole de La Forge, Noël Parfait, Camille Pelletan, Antonin Proust. S'y rencontraient aussi des historiens (Alfred Rambaud), des archivistes (Jules Guiffrey) et diverses personnalités attachées au Ministère de l'Instruction publique (Louis Liard). Les arts étaient représentés par le peintre François Flameng, par le directeur des Musées nationaux, Albert Kaempfen, le directeur des Beaux-Arts, Gustave Larroumet. Quant à Charles-Louis Chassin, Adrien Duvand et Ernest Hamel, ils appartenaient au monde de la presse ${ }^{8}$. Si, par rapport à 1888 , quelques absences, celle de Champfleury par exemple, s'expliquent par la mort, celle de Dalou paraît plus étonnante. En 1890, la SHRF comptait 130 membres, qui payaient une cotisation annuelle de 20 francs $^{9}$; Charavay espérait que le nombre des sociétaires allait augmenter et fit appel au 
dévouement de tous les adhérents pour assurer « la propagande nécessaire ${ }^{10}$. En 1890, Aulard incita à son tour les membres à recruter des adhérents, car, fit-il observer, "plus nous serons nombreux, plus intéressante et féconde sera notre œuvre $~^{11}$ À l'occasion du Centenaire, la Société organisa au Louvre une exposition sur la Révolution ${ }^{12}$, qui produisit un bénéfice de plus de 17000 francs; sa situation financière était donc saine ${ }^{13}$.

Lors de l'assemblée du 2 mars 1890, Alphonse Aulard prononça un long discours pour exposer les buts de la Société et présenter son activité intellectuelle et éditoriale. « $\mathrm{Ce}$ que voulaient les fondateurs, dit-il, c'était avant tout faire œuvre de science. Il nous semblait qu'il était grand temps de ne plus traiter l'histoire de la Révolution comme une matière à pamphlet et d'appliquer à cette histoire les mêmes règles de critique scientifique qu'aux périodes les plus anciennes ${ }^{14} »$. D'après Aulard, un obstacle majeur s'opposait auparavant à cet idéal : il manquait «la condition indispensable à toute science, je veux dire la liberté. Ce n'est que depuis l'établissement définitif de la République qu'il est permis de raconter avec pleine franchise notre grande crise nationale et de la juger comme on veut ${ }^{15} »$. Une autre modification essentielle était intervenue. Journalistes, écrivains, parlementaires: plus personne ne cherchait désormais à se draper "dans les habits des aïeux »: "L'esprit de la Révolution n'est plus enfermé dans les formes révolutionnaires. On essaie de continuer la Révolution, on n'en singe pas les gestes. Il s'ensuit que l'historien peut aujourd'hui critiquer à son aise Vergniaud, Robespierre ou Danton, sans qu'aucun des partis ou des hommes de notre temps puisse s'en plaindre ou s'en féliciter comme d'une allusion malveillante ou flatteuse ${ }^{16}$ ». Pour Aulard, l'histoire de la Révolution devait échapper " aux légendes, aussi bien de la gauche que de la droite » et s'écrire " comme s'il s'agissait du règne de Philippe-Auguste ou de Louis XIV ». En servant ainsi la science, estimait Aulard, les membres de la Société servaient aussi la République, car la science entraîne le recul du "préjugé théocratique » et sert la cause des esprits libres, « c'est-à-dire la République et la religion des cœurs généreux, c'est-à-dire la patrie $»^{17}$.

5 Dans ce discours, Aulard fixa quelques règles de comportement entre sociétaires. Ses membres devaient bannir « les fausses camaraderies » et ne pas se sentir liés par une solidarité de mauvais aloi. "Aucun de nous, dit-il, n'a abdiqué [le droit] de critiquer, avec une courtoise fermeté, les écrits de ses confrères et on peut nous rendre cette justice que nous ne sommes pas une société d'admiration mutuelle ${ }^{18}$ ».

Un dernier point mérite d'être souligné : la Société devait encourager particulièrement les historiens de province qui se heurtaient à de grandes difficultés pour faire connaître les fruits de leurs recherches : «À ces savants isolés, à ces travailleurs aussi négligés que militants, nous offrons un point de ralliement et ils ont l'espérance que notre Société pourra, dans la limite de ses modestes ressources, les aider à publier leurs travaux ${ }^{19}$ ». Aulard signala encore que, si la Société ne pouvait, faute de moyens, se lancer elle-même dans de grandes entreprises éditoriales, en revanche plusieurs de ses membres étaient associés à la publication de grandes collections lancées sous l'égide du Conseil municipal de Paris ou du Ministère de l'Instruction publique. Ainsi, avait-il entrepris lui-même la constitution du Recueil des actes du Comité de Salut public, tandis qu'Étienne Charavay s'était lancé dans l'édition de la correspondance générale de Carnot ${ }^{20}$. Malgré le manque de moyens, la Société avait d'ailleurs assuré elle-même une édition critique de la célèbre brochure de Sieyès, avec variantes et commentaires, publiée par Edme Champion et édité l'étude prosopographique que Jules Guiffrey avait 
consacrée aux membres de la Convention. D'autres travaux étaient encore envisagés, comme la publication de la liste critique des membres des Assemblées révolutionnaires par Étienne Charavay et Louis Frédéric Tuetey.

\section{La Société d'histoire moderne}

7 Une dizaine d'années plus tard, divers membres de la SHRF, et non des moindres, comptèrent parmi les promoteurs d'une nouvelle société, dont le champ de recherches incluait la période révolutionnaire, tout en étant chronologiquement beaucoup plus large. La "première séance provisoire" de la SHM se déroula le 22 juillet 1901 ; auparavant, un certain nombre d'historiens avaient reçu une circulaire dont la Revue de synthèse historique reproduisit un extrait; jusqu'alors, constatait ce texte, à cause, notamment, de la difficulté de s'abstraire "des passions contemporaines", il avait semblé que "l'histoire moderne » offrait « un champ plus propre aux développements littéraires, dramatiques ou philosophiques, qu'aux investigations des historiens». Pourtant, cette histoire était en train de s'élaborer, en partie grâce à la SHRF et de la Revue d'histoire moderne et contemporaine, fondée en 1899; le moment semblait donc venu, pour "tous les érudits voués aux mêmes études ", d'appliquer "une commune méthode, d'en faire reconnaître la valeur et la nécessité, d'en répandre l'usage pour les périodes historiques dont ils s'occupent depuis le XVI siècle jusqu'à nos jours ${ }^{21}$. Au nom du Comité d'initiative, Albert Mathiez exposa le but de la nouvelle Société, qui était de "remédier à l'état d'isolement des historiens de l'époque moderne"; aux pratiques des historiens, adeptes « du principe du chacun chez soi ${ }^{22}$, il opposa celles des physiciens, des biologistes ou des humanistes, accoutumés à se réunir pour s'entretenir de leurs travaux. Grâce à la SHM, dit Mathiez, les chercheurs isolés pourraient désormais se rencontrer, à condition toutefois de respecter une exigence fondamentale : ne pourraient être reçus membres que les historiens appliquant « dans leurs travaux la méthode rationnelle", ce qui rendait impossible l'adhésion de "quiconque étudie le passé avec des idées préconçues, quiconque y cherche des arguments en faveur de ses doctrines politiques ou confessionnelles, quiconque apporte dans l'histoire l'esprit de parti et d'exclusivisme $»^{23}$. L'activité essentielle de la Société consisterait en réunions mensuelles durant lesquelles seraient présentées des communications, dans un esprit qui devrait évoquer non pas les travaux d'une académie, mais ceux "d'un atelier de recherches". La vérité étant la première préoccupation de la Société, toutes les critiques et toutes «les conceptions nouvelles » devaient être formulées " avec une entière indépendance »; Mathiez se disait persuadé qu'aucun sociétaire «ne se froissera[it] d'être rectifié, critiqué même par un de ses confrères ${ }^{24}$. Deux fois par an, à Pâques et durant les grandes vacances, se déroulerait une assemblée générale, prioritairement réservée aux communications des provinciaux ${ }^{25}$. Enfin, le Bureau devait entretenir une correspondance suivie avec les membres ne résidant pas à Paris, les mettre en rapport avec leurs confrères parisiens et leur fournir des renseignements. Au terme de cet exposé, l'assemblée acclama trois présidents d'honneur : Alphonse Aulard, Ernest Lavisse et Gabriel Monod. Un bureau provisoire fut constitué, composé d'Émile Bourgeois, président, d'Henry Lemonnier, vice-président, d'Albert Mathiez, secrétaire général ${ }^{26}$, de Pierre Muret, secrétaire adjoint et de Camille Bloch, archiviste et trésorier. Pierre Muret présenta les statuts provisoires, rédigés sur la base de ceux de la Société de physique, fondée en 1873, «dont le fonctionnement, jusqu'à ce jour, a été des plus réguliers ${ }^{27}$. Pour éviter les 
coteries et assurer le fonctionnement démocratique de la Société, le président ne pouvait pas être réélu à la fin de son mandat, et le vice-président accédait automatiquement à la présidence. Un Comité de présentation examinait les candidatures ; les adhérents devaient payer une cotisation annuelle - de dix francs pour les Parisiens, de cinq francs pour les provinciaux - incluant le prix du bulletin mensuel, qui se présentait comme "un résumé très succinct " des séances ${ }^{28}$. Enfin, étaient prévues la publication de textes sous la direction et aux frais de la Société, la création "d'un Bureau international de correspondance historique » et la formation de groupes d'étude en province ${ }^{29}$. Quelques mois après ces dispositions provisoires, le 31 octobre 1901, se tint la première séance générale, qui vit la confirmation du bureau provisoire. La Société comptait alors 103 membres, essentiellement des universitaires et des professeurs de lycée ; sur la liste des adhérents, se détachent un recteur d'Académie, un inspecteur général, trois archivistes, des directeurs de revues, comme Henri Berr, directeur de la Revue de synthèse historique, fondée en 1900, Georges Bourgin, Gaston Brière et Pierre Caron de la Revue d'histoire moderne, ou encore François Picavet, de la Revue internationale de l'enseignement, publiée depuis 1881 ; figuraient aussi un avocat de la Cour d'appel et quelques étudiants. La plupart des sociétaires résidaient à Paris ou dans la banlieue parisienne, trente vivaient en province, et un Italien habitait Frascati 30 .

Comme la SHRF, la SHM se voulait attentive aux recherches se déroulant en dehors de la capitale. En 1902, dans son bulletin, fut publié un rapport de Pierre Caron relatif à "l'organisation des études locales d'histoire moderne " $^{31}$; en 1904, elle rédigea « une instruction destinée à faciliter les travaux d'histoire moderne en province $»^{32}$ comprenant des conseils pratiques et des suggestions de sujets à traiter. Elle se montrait encore soucieuse de l'élaboration des sources utiles aux historiens; c'est pourquoi elle suggéra aux présidents du Sénat et de la Chambre des députés d'» améliorer le plan et le mode de publication des Archives parlementaires ${ }^{33}$. Elle était attentive au statut et à l'organisation des archives: le 6 mars 1904, Henry Lemonnier annonça avoir reçu communication d'une proposition de loi "portant réorganisation générale des Archives de France ", dont la rédaction, dit-il, avait été « empruntée » à Ernest Coyecque, archiviste-paléographe, membre de la Société ${ }^{34}$; en 1908, elle s'inquiéta de récents décrets, signés à l'instigation de Philippe Berthelot, visant à restreindre la communication des archives du Ministère des Affaires étrangères ${ }^{35}$. Soucieuse des conditions de travail de ses membres, elle souhaitait une réorganisation de la Bibliothèque dite "des Sociétés savantes » et préconisait le prêt des ouvrages possédés en double, qui pourraient constituer une "bibliothèque circulante ». Elle espérait « constituer un centre de discussion et d'information », créer une Bibliothèque d'histoire moderne comparable aux Bibliothèques allemandes Bertrage ou Sammlungen, et publier «des brochures à bon marché relatives à des questions politiques, administratives, sociales de la France depuis $1789 \Perp^{36}$. Selon Pierre Caron, la Société tenait à conserver " son originalité de groupement purement scientifique » tout en continuant à "être sévère à la mauvaise foi, au mépris et à l'ignorance de la méthode » et à « créer toujours plus de solidarité entre les travailleurs, à accroître le nombre et la valeur de leurs moyens d'information et de recherche ». Enfin, elle était soucieuse « de développer les relations entre historiens français et historiens étrangers, et de ménager ainsi la possibilité de ces enquêtes internationales dont, pour une foule de sujets, on peut attendre des résultats si féconds $»^{37}$. 
9 Toujours d'après Pierre Caron, les qualités exigées par la Société pour l'admission des candidats limitaient sa croissance, mais, à l'inverse, l'étendue chronologique du champ des études, allant des Guerres d'Italie jusqu'au début du XXe siècle, et le souci de construire une " histoire intégrale, histoire littéraire et histoire de l'art comprises " $^{38}$ pouvaient accroître le nombre des postulants. En 1904, la SHM comptait 159 membres et Pierre Caron n'imaginait pas que ce nombre fût jamais "très élevé »; une telle éventualité ne lui paraissait d'ailleurs pas souhaitable, une augmentation du nombre des adhérents étant susceptible de «troubler l'homogénéité» du groupe; contrairement à ce qui se passait à la SHRF, il recommandait «d'attendre les candidatures plutôt que de les susciter ${ }^{39}$. Sur les 159 adhérents, 86 étaient professeurs à l'Université ou dans des établissements secondaires ; comme l'écrit Caron, rien n'était fait pour attirer dans la Société « les historiens-publicistes, fournisseurs ordinaires des journaux et des revues à grand tirage ». Les hommes politiques n'étaient pas mieux accueillis que les journalistes et si "quelqu'un » figurait dans la liste des membres, c'était «au titre, incontestable, d'historien " ${ }^{40}$. La présentation des sociétaires se clôt sur un ton féroce: "Celle-ci [la Société d'histoire moderne], dès sa naissance, a joui d'une réputation d'exclusivisme, qu'ont accrue quelques exécutions de mauvais livres. Ses exigences en matière de méthode et la juste sévérité de ses arrêts étaient de nature à rebuter amateurs et journalistes ; aussi les candidatures de cet ordre ne se sont-elles pour ainsi dire pas produites ${ }^{41} »$. En 1902, une discussion s'était élevée autour de la candidature de Nathanaël Weiss, qui ne postulait pas à titre personnel, mais comme représentant de la Société d'histoire du protestantisme français, dont il était le bibliothécaire, et qui ne fut pas admis comme membre. Deux raisons motivèrent ce refus: le caractère «exclusivement protestant» de la Société d'histoire du protestantisme français et le fait qu'il s'agissait d'une candidature collective ${ }^{42}$.

En 1902 et en 1904, le bulletin de la Société présenta un classement des adhérents par spécialités. La grande majorité des travaux étaient confondus dans une vaste catégorie, bien significative, regroupant "l'histoire politique, diplomatique, religieuse et militaire "; en 1904, pour cette rubrique, étaient cités 89 noms $^{43}$; suivaient l'histoire économique et sociale, avec 26 noms, l'histoire de pays étrangers, représentée par 25 personnes et l'histoire locale par 18; la littérature et la philosophie étaient représentées par 19 membres, les beaux-arts par 10, l'histoire de l'enseignement, l'histoire coloniale et la spécialisation bibliographique étant illustrées par moins de cinq noms chacune. Entre 1901 et 1904, la Société entendit trente-quatre communications. Le plus grand nombre de ces communications avaient porté sur «la méthode et [...] l'organisation du travail historique $»^{44}$; pour Pierre Caron, elles avaient présenté un intérêt plus grand que les autres interventions, consacrées à des points d'histoire précis.

11 C'est précisément la question de la méthode historique qui agita la vie de la société en 1903. Le 3 janvier, François Simiand présenta une communication intitulée «Méthode historique et science sociale, étude critique d'après les ouvrages récents de $\mathrm{M}$. Seignobos et de M. Lacombe ", fondée sur un jugement sévère sur des livres des deux auteurs cités. Selon Simiand, les études historiques devaient recevoir une nouvelle orientation et trois «idoles » être combattues : l'idole politique, l'idole individuelle et l'idole chronologique. "En résumé, dit-il, il faudrait choisir entre l'étude de l'individuel, du contingent, sans ordonnance réfléchie, sans méthode pleinement consciente, et l'étude positive, objective du phénomène humain susceptible 
d'explication scientifique, tendant à l'élaboration consciente d'une science sociale ${ }^{45}$ ». Présent, Seignobos réagit, semble-t-il, avec un certain détachement: il déclara qu'il n'avait jamais nié qu'on pût "arriver à établir des lois des phénomènes sociaux » et qu'il ne se trouvait « donc pas en désaccord avec M. Simiand » ${ }^{46}$.

À la fin de l'année 1903, se posa un problème d'une autre nature; le bulletin du mois de décembre mentionne "la reprise de la discussion née lors de la dernière réunion autour d'une candidature $\aleph^{47}$. Lors de la réunion du 6 décembre, le président Lemonnier expliqua que, pour les membres du Bureau et du Comité de présentation, les seules candidatures devant être écartées étaient celles «d'historiens qui auraient manqué, dans leurs travaux, aux règles de la méthode ou de la probité scientifique ou qui, adversaires déclarés des idées modernes, les auraient combattues publiquement ou par des actes »; la Société, ajouta Henry Lemonnier, devait s'ouvrir «à toutes les bonnes volontés et aux travailleurs méritants ». Mais certains adhérents, ajouta-t-il, voulaient qu'elle fût «un groupement très restreint de membres, en absolue conformité d'opinions, non seulement scientifiques, mais surtout politiques, comme un cercle d'amis plutôt fermé $»^{48}$. Aussi, les membres du Bureau et du Comité démissionnèrent collectivement ${ }^{49}$. En 1904, Pierre Caron constata que "les exigences intellectuelles, notamment celles qui se rapportaient au rationalisme", ne suffisaient pas "pour assurer une identité des idées politiques et sociales au sein de la société » et que les "sympathies sentimentales" de certains historiens "rompus à la pratique de la méthode, bien informés et sachant travailler » se trouvaient "à droite ». Aussi, dit-il, il s'était formé "une gauche et, sinon une droite, un centre", facteur qui jouait aussi pour l'admission ou le refus de nouveaux adhérents, les membres de gauche souhaitant pratiquer une politique d'adhésion restrictive, pour des raisons d'ordre idéologique ; à l'époque où il écrivait, la position dominante était celle du président Lemonnier, désireux d'» entrebâiller le plus largement la porte ». Cet entrebâillement permit à la Société d'atteindre le nombre de 200 adhérents - parmi lesquels on relève les noms d'Abel Ferry et de Joseph Reinach ${ }^{50}$ - en septembre 1909.

\section{La Société d'histoire de la Révolution de $1848^{51}$}

Parmi les périodes étudiées par les membres de la SHM, l'une retenait plus particulièrement l'attention de deux sociétaires: la Deuxième République. L'idée de réserver un sort particulier à 1848 vint tout d'abord de Georges Renard. Le 10 mai 1903, celui-ci présenta à ses confrères de la SHM une communication intitulée "De l'utilité qu'il y aurait à créer une section spéciale pour l'étude scientifique de la Deuxième République française $~_{52}$; d'après lui, les années 1848-1851 représentaient « un moment de haute importance dans l'histoire du XIXe siècle », correspondaient au passage " d'un grand mouvement idéaliste » à " un grand mouvement réaliste»; en politique, elles avaient vu s'accomplir "un grand pas vers la démocratie », sous l'angle de l'histoire sociale, elles avaient marqué le point de départ de la législation du travail, et sous celui de l'histoire économique, elles avaient préludé à la législation industrielle. "Tous les problèmes actuels ", dit Georges Renard, avaient été posés par la Deuxième République, qui avait tenté de les résoudre, qu'il s'agit des rapports entre l'État et les Églises, de l'arbitrage dans les conflits du travail, du rachat des chemins de fer, des relations internationales, etc. Et pourtant, poursuivit Renard, seuls « un petit nombre de livres » relatifs à cette période avaient été publiés et «de vastes espaces" restaient «à 
défricher ». Georges Renard avait soigneusement élaboré son projet, fixé la périodicité des réunions, à raison de deux par an, et établi un plan de recherches; il avait ainsi distingué cinq types d'évolution à étudier (technique et économique, politique, religieuse et philosophique, morale, scientifique, artistique et littéraire). Il pensait que cette spécialisation pourrait s'appliquer à d'autres époques et, qu'ainsi, la SHM, «sans cesser d'être une, deviendrait de plus en plus, par la diversité de ses sections, un grand organisme très vivant et très actif, qui rendrait de précieux services à la science et aux savants $»^{53}$. Son projet fut appuyé par Ernest Denis, pour qui il n'existait que «des travaux de dilettante » sur 1848, et par Pierre Caron, qui voyait là l'occasion d'attirer l'attention sur des archives accessibles aux lecteurs ${ }^{54}$. Il fut vivement combattu par Léon Cahen et Henry Lemonnier; le premier estimait que les membres s'occupant des mêmes questions ou des mêmes périodes pouvaient parfaitement se connaître et correspondre sans créer de sections spéciales, processus dangereux pour l'unité de la société ; quant au second, il jugeait préférable de laisser les membres se regrouper spontanément, quitte à décider plus tard si, oui ou non, la création de sections particulières était nécessaire. Pour Gilles Candar, ce refus peut s'expliquer par les difficultés que la Société connaissait en cette année 1903, par la crainte d'un intérêt trop marqué pour les problèmes d'actualité et par la personnalité de Georges Renard, que sa carrière atypique plaçait en retrait du monde universitaire, et dont on craignait l'autoritarisme.

Un autre homme nourrissait un vif intérêt pour la Révolution de 1848 et la Seconde République : Henry Michel ${ }^{55}$. En 1903, il prononça une conférence sur Edgar Quinet, à Lyon ${ }^{56}$ et s'apprêtait à publier son étude sur la Constitution de $1848^{57}$. Comme Georges Renard, Henry Michel estimait que l'histoire de 1848 aidait à mieux comprendre la Troisième République; il déplorait qu'aucune histoire sérieuse de cette période n'existât encore et attendait tout d'abord des monographies et « des études critiques sur des points très limités ${ }^{58}$, qui permettraient d'élaborer, ultérieurement, des études générales et des synthèses. Par ailleurs, il estimait que les jeunes gens devaient connaître 1848; se référant au chancelier d'Aguesseau, il jugeait étonnant que la jeunesse entendît toujours parler des faits «les plus reculés», mais jamais des plus récents ${ }^{59}$.

Henry Michel était, lui, favorable à la création d'une société indépendante, formée sur le modèle de la SHRF. Durant l'été 1903, il se tourna vers Georges Renard, qui reconnut bien volontiers que, sans lui, son propre projet serait resté «à l'état de rêve " ${ }^{60}$. Pour préserver le caractère scientifique de l'entreprise, Henry Michel souhaitait éviter une approche militante et s'adresser essentiellement à des historiens: "Pour donner l'impression d'une société scientifique, disait-il, il faut éviter d'être ou de paraître submergé par l'élément politique ». Pour autant, il ne voulait pas décourager la bonne volonté des parlementaires; en homme pragmatique et avisé, il pensait que l'élément politique pouvait être utile " pour la propagande d'idées ", mais aussi, «à un point de vue très réaliste ", pour obtenir des subventions.

Un comité d'initiative fut formé comprenant, outre Henry Michel et Georges Renard, Alphonse Aulard, Antonin Debidour, Ernest Denis, Maurice Faure, Gustave Geffroy et diverses autres personnalités. Une réunion préliminaire se déroula chez Henry Michel et fut suivie de 1 'Assemblée générale constitutive, tenue à la Sorbonne, amphithéâtre Edgar Quinet, le 24 février 1904; Adolphe Carnot, frère de Sadi Carnot et président de l'Alliance républicaine démocratique, y prononça une allocution et souligna 
l'importance du «mouvement d'idées des plus remarquables parmi les penseurs », qui s'était produit entre 1830 et 1840 , avant de se poursuivre "plus tard dans les masses populaires en dépit de toutes les mesures de coercition prises par le Second Empire ${ }^{61}$.

17 Le premier Bureau comprenait huit personnes: Adolphe Carnot, président ; Alphonse Aulard, Maurice Faure et Alexandre Millerand, vice-présidents; Henry Michel, secrétaire-général et Pierre Caron, secrétaire général adjoint ; Camille Bloch, trésorier ; Georges Renard, rédacteur du bulletin, qui était conçu comme «la véritable cheville ouvrière » ${ }^{62}$ de la Société. Fut aussi nommé un Comité directeur de trente-deux membres, dans lequel siégeaient, outre des historiens - Antonin Debidour et Charles Seignobos, par exemple -, des représentants d'autres disciplines, comme Charles Gide, des écrivains, entre autres Anatole France et Lucien Descaves ; le monde politique était représenté par des socialistes (Jean Jaurès, Victor Augagneur), des radicaux (Léon Bourgeois); deux "veuves républicaines", si l'on ose dire, Mesdames Paul Bert et Charles Floquet, rappelaient la République du temps de Jules Ferry et de Gambetta.

Peu après la tenue de l'assemblée constitutive, le 10 mars 1904, Henry Michel déposa à la préfecture de police les statuts, calqués sur ceux de la SHRF; d'après l'article 2 , la SHR de 1848 avait pour objet « de grouper les personnes qui, en tous pays, s'intéressent dans un esprit démocratique, à l'histoire de cette époque [1848], soit en France, soit à l'étranger, et d'en organiser l'étude scientifique »; d'après l'article 3, elle devait «faire office de bureau d'information pour les historiens qui s'occupent de cette époque ».

Dès 1904, la Société compta 180 membres et sa composition la distinguait quelque peu des deux sociétés précédentes ${ }^{63}$. Alors que celles-ci étaient exclusivement masculines, elle comptait en son sein sept personnes du $\operatorname{sexe}^{64}$; y figuraient aussi deux ecclésiastiques, Mgr Péchenard, recteur de l'Institut catholique de Paris, et l'abbé Toiton $^{65}$, tandis qu'aucun ministre du culte catholique ne semble avoir appartenu à la SHRF ou à la SHM ; enfin, contrairement à la SHM qui avait refusé l'adhésion de Nicolas Weiss, la SHR de 1848 accepta celle d'un habitant de Villeneuve-sur-Lot présentée au nom de la Jeunesse laïque de cette ville et celle, collective, de la section de la Ligue des droits de l'homme d'Angoulême. Trente-quatre adhérents résidaient en province et quatre étaient étrangers. Certains noms d'adhérents sont intéressants à relever, pour des raisons diverses, comme ceux de François Arago, d'Armand Barbès, fils du grand Barbès, de Léon Blum, François Carnot, Lucien Herr, des frères Margueritte, de Romain Rolland, et de bien d'autres encore. En 1905, la liste des sociétaires s'était accrue d'une vingtaine de membres; en outre, une petite dizaine de bibliothèques françaises (Bordeaux, Dijon, Le Mans.) ou étrangères (Lausanne, Berlin.), ainsi que les Archives départementales du Cantal s'étaient abonnées à La Révolution de 1848 ${ }^{66}$. En 1907, le nombre des adhérents s'élevait à $255{ }^{67}$. À cette date, Henry Michel -mort le 13 décembre 1904 - avait été remplacé par Hippolyte Monin, puis par Georges Renard.

Comme les deux sociétés précédentes, la SHR de 1848 était doublement attentive à la province, que ce fût par les sujets traités dans la revue, par exemple le suffrage universel dans le département du Lot, les élections de 1848 dans le Loiret, la situation dans l'Ain, etc., ou par l'attention portée aux sociétaires provinciaux, conviés avec insistance à assister aux assemblées générales et à y présenter une communication, si possible « courte et frappante $»^{68}$.

21 Des liens forts et des affinités étroites unissaient les trois sociétés. Les triples appartenances étaient nombreuses, de la part des historiens connus, mais aussi de membres plus obscurs. Les sociétés s'informaient sur leurs activités respectives ; ainsi, 
en 1904, le BSHM reproduisit l'annonce que Georges Renard avait faite lors de la réunion du mois de mars, relativement à la fondation de la SHR de 1848 ; Renard avait souligné la présence de plusieurs membres du Bureau de la SHM au sein du Bureau de la SHR de 1848, estimant que cela indiquait bien «la pensée de sincère confraternité qui anim[ait] les membres de la Société nouvelle ${ }^{69}$. En février 1907, Aulard, présent au banquet de la SHR de 1848, loua la méthode de travail des spécialistes de la Deuxième République, fondée sur "une patiente recherche et une consciencieuse étude de documents originaux $»^{70}$; en mars 1908, Émile Bourgeois représenta la SHM au banquet organisé par la SHRF; il prononça un discours, insista sur "l'étroite parenté » et "l'esprit de famille » unissant les deux organismes et souligna « la manière commune de travailler selon les bonnes règles de la méthode historique $»^{71}$. En janvier 1908, après la signature des décrets restreignant l'accès aux archives du Ministère des Affaires étrangères, les trois sociétés firent des démarches communes pour tenter d'atténuer les effets de ces textes; deux autres sociétés se joignirent d'ailleurs à elles: la Société d'histoire diplomatique et la Société d'histoire contemporaine ${ }^{72}$.

Malgré tout, la SHM semblait animée par un certain sentiment de supériorité scientifique ; ainsi, en 1904, Pierre Caron remarqua avec une sorte de dédain, semble-til, que la SHRF et la SHR de 1848 avaient "par destination une couleur politique » et que « des hommes politiques figur[aient] dans leur bureau ». Cette réserve est d'autant plus remarquable que ces sociétés puisaient dans les mêmes viviers politiques, chez les socialistes, les radicaux, les dreyfusards et les républicains laïques ${ }^{73}$. Les réticences de Pierre Caron ne l'empêchaient toutefois pas de suggérer la formation d'une fédération des "Sociétés d'histoire moderne ", qui interviendrait utilement dans les rapports avec "les cercles savants de l'étranger » et permettrait aussi «d'établir en commun une salle de lecture avec livres et revues", étant entendu que chaque société fédérée conserverait son autonomie. Cette fédération serait la «manifestation décisive, dans le groupe des historiens rationalistes, de cet esprit de solidarité, indispensable au progrès de la Science, qu'il faut propager par le conseil et, mieux encore, par l'exemple ».

Ce que nous venons d'exposer ne correspond qu'à une partie des échanges établis entre les historiens de cette époque ; une approche plus globale appellerait l'étude de liens établis grâce à d'autres sociétés ou associations, savantes ou non - on pense essentiellement à la Ligue des droits de l'homme, à la Ligue de l'enseignement et à la franc-maçonnerie - et à d'autres revues, notamment la Revue de synthèse historique, la Revue internationale de l'enseignement, citées plus haut, mais aussi la Revue de métaphysique et de morale, dont certains collaborateurs appartenaient à l'une ou l'autre des sociétés étudiées. Ce travail de fond ne pouvait toutefois se faire dans le cadre d'une communication à laquelle, inévitablement, des limites ont été assignées.

\section{NOTES}

1. Les dossiers des sociétés reconnues d'utilité publique peuvent être consultés dans les locaux du Bureau des associations reconnues d'utilité publique, 50 avenue Daumesnil, 75012 Paris. Voir aussi l'important article « Société », Grande encyclopédie, t. 30, p. 152. 
2. Il conviendrait d'analyser attentivement les usages des mots « moderne » et " contemporain », qui, en France, ne furent pas toujours distingués aussi rigoureusement qu'ils le sont actuellement par les historiens (comme on le sait, les historiens français adoptent des limites chronologiques qui leur sont propres). Ainsi, en 1898, le président de la Société d'histoire contemporaine mentionnait "cette partie de l'histoire moderne qui se qualifie de contemporaine", Société d'histoire contemporaine, Assemblée générale de 1898, Discours du Président [comte Alfred Boulay de la Meurthe], Besançon, imprimerie Jacquin, 1898, p. 1. Mutatis mutandis, l'expression d'» histoire contemporaine " pourrait avoir eu, pour les «modernistes » de l'époque, la même valeur que celle d'« histoire du temps présent » pour les « contemporanéistes » actuels. La Société d'histoire contemporaine, qui recrutait dans les milieux catholiques, se considérait comme une société pionnière en matière de travaux consacrés aux temps les plus proches; en 1898, son président rappela que, traditionnellement, les érudits considéraient que les travaux sur la période la plus récente de l'histoire «n'étaient qu'un produit de l'imagination et de l'esprit de parti, qu'un plaidoyer mêlé de roman », ibid.

3. Louis HALPHEN, "France", Histoire et historiens depuis cinquante ans. Méthode, organisation et résultats du travail historique de 1876 à 1926, Paris, Librairie Félix Alcan, 1927, tome I, p. 152. À ce travail, il convient d'ajouter un précieux tableau intitulé « Les historiens français : la constitution d'une discipline académique. 1800-2005 ", sur le site de l'INRP http://www.inrp.fr/she/nhist/ scripts/affichage.php?table\%20=associations

4. Société de l'histoire de la Révolution française [SHRF], Compte rendu de l'assemblée générale du 2 mars 1890, Paris, au siège de la Société, 1890, p. 8.

5. SHRF, Célébration historique du centenaire de 1789, catalogue des objets formant l'Exposition historique de la Révolution française, Paris, au siège de la Société, 1889, p. IV

6. SHRF, Compte rendu de l'assemblée générale du 2 mars 1890..., op. cit., p. 7.

7. ibid., p. 24.

8. SHRF, Célébration historique du centenaire de 1789..., op. cit., p. III.

9. À la Société de l'histoire de France, la cotisation était de 30 francs, à la Société d'histoire du protestantisme, de 10 francs et à la Société des études juives, de 25 francs, Grande encyclopédie, s.v. «Société », t. 30, p. 152.

10. SHRF, Compte rendu de l'assemblée générale du 2 mars 1890..., op. cit., p. 30.

11. Ibid., p. 26.

12. Cette exposition dura du 18 avril au 17 novembre 1889 ; elle put se tenir au Louvre grâce à l'appui du ministre de l'Instruction publique et du directeur des Beaux-Arts. Durant les années précédant le Centenaire de la Révolution, Charles-Louis Chassin et Étienne Charavay avaient envisagé la création d'un Musée de la Révolution, qui aurait été établi dans le jardin des Tuileries, mais ce projet n'aboutit point, Étienne CHARAVAY, Le Centenaire de 1789 et le Musée de la Révolution, Paris, Charavay Frères, 1886, p. 28-31.

13. SHRF, Compte rendu de l'assemblée générale du 2 mars 1890..., op. cit., p. 28.

14. Ibid.,p.12.

15. Ibid.

16. Ibid.

17. Ibid.,p.13.

18. Ibid., p. 14.

19. Ibid., p. 15.

20. Ouvrage dont Mathiez, à une époque où il n'était pas encore fâché avec Aulard avait souligné l'importance, voir Albert MATHIEZ, « M. Aulard historien et professeur », Revue des Cha-rentes, 31 mars 1905; Aulard publia lui-même cet article (Révolution française, juillet-décembre 1908, p. 46-60), pour montrer l'évolution du jugement de Mathiez sur sa personne et son œuvre. Information communiquée par Claude Mazauric.

21. Revue de synthèse historique, $\mathrm{n}^{\circ}$ 7, août 1901, p. 106. 
22. Bulletin de la Société d'histoire moderne [BSHM], juillet 1901, p. 1.

23. ibid.

24. ibid.

25. En 1902, Mathiez souhaita que des démarches fussent entreprises auprès des compagnies ferroviaires dans le but d'obtenir un tarif réduit pour les membres provinciaux, BSHM, décembre 1902, p. 65. Cependant, en 1907, Émile Bourgeois fit remarquer que l'assemblée générale de Pâques attirait peu de provinciaux, et que, par ailleurs, elle ne retenait qu'un petit nombre de Parisiens, BSHM, décembre 1907, p. 5.

26. Nommé professeur au lycée de Châteauroux, Albert Mathiez démissionna rapidement ; il fut remplacé par Charles Andler, ibid., décembre 1901, p. 13.

27. Ibid., juillet 1901, p. 3. Faut-il voir dans cet emprunt la marque d'un simple souci de la bonne marche administrative de la Société ? Ne traduirait-il pas plutôt la volonté d'affirmer symboliquement le caractère scientifique de l'histoire?

28. Ibid., p. 4.

29. Ibid.

30. Ibid., novembre 1901, p. 9-11. Les provinciaux venaient d'une vingtaine de villes différentes, quelques-unes comptant plusieurs membres (Lille et Orléans : 4 ; Lyon, Marseille et Toulouse :2).

31. Ibid., mai 1902, p. 41-52.

32. Pierre CARON, La Société d'histoire moderne (1901-1904), extrait de la Revue de synthèse historique, Paris, Léopold Cerf, 1904, p.5. Ce texte fut publié la Revue de synthèse historique, avril 1904, p. 243-249.

33. BSHM, mai 1902, p. 52.

34. Ibid., mars 1904, p. 122 (cette proposition de loi fut présentée par Gabriel Deville et Paul Barthou, le 8 février 1904).

35. Ibid., janvier 1908, p. 13.

36. Pierre CARON, La Société d'histoire moderne (1901-1904)..., op. cit., p. 5.

37. ibid.,p. 6

38. ibid.,p. 2.

39. Ibid.

40. Ibid.

41. Ibid.

42. BSHM, avril 1902, p. 30.

43. Cette catégorie était elle-même divisée en plusieurs champs chronologiques; le plus grand nombre de travaux cités se rapportent à la Révolution française (23), à la période contemporaine (18), à l'Empire (14) et au XVIII ${ }^{\mathrm{e}}$ siècle (13). Un même nom pouvait figurer dans plusieurs rubriques.

44. Pierre CARON, La Société d'histoire moderne (1901-1904)..., op. cit., p. 3.

45. BSHM, janvier 1903, p. 76. Cette communication de François Simiand correspond à son célèbre article, publié par la Revue de synthèse historique, janvier-avril 1903, p. 129-157. Pour Jacques Revel, les critiques émises par François Simiand ne traduisent pas seulement un conflit méthodologique et épistémologique, mais aussi une rivalité entre disciplines académiques, Jacques REVEL, « Histoire et sciences sociales », Mil neuf cent, Comment on se dispute, $n^{\circ} 25,2007 / 1$, p. 101126. Selon Madeleine Rébérioux, ce débat, d'ordre institutionnel, politique et épistémologique, était aussi marqué " par des tensions de générations », Madeleine REBÉRIoux, «Le débat de 1903 : historiens et sociologues ", Au berceau des Annales. Le milieu strasbourgeois. L'histoire en France au début du XXe siècle, Charles-Olivier CARBonell et Georges Livet dir., Toulouse, Presses de l'IEP 1983, p. 222.

46. BSHM, janvier 1903, p. 76.

47. Ibid., décembre 1903, p. 106. Le Bulletin ne mentionne pas de discussion de cette nature lors de la réunion de novembre. 
48. BSHM, décembre 1903, p. 106.

49. Les membres démissionnaires étaient, pour le Bureau : Henry Lemonnier, Édouard Driault, Charles Schmidt et Camille Bloch (Ernest Denis, vice-président, avait démissionné antérieurement, pour raison de santé) et pour le Comité de présentation : Alphonse Aulard, Émile Bourgeois, Antonin Debidour, Henri Hauser et Gustave Lanson, BSHM, décembre 1903, p. 106. Des élections eurent lieu en janvier 1904 ; tous les membres démissionnaires furent réélus, Ernest Denis étant toutefois remplacé par Debidour à la vice-présidence et Seignobos prenant la place de celui-ci au Comité de présentation, BSHM, janvier 1904, p. 110.

50. Société d'histoire moderne, Table et Bulletin. Liste des membres, Paris, Édouard Cornély, 1909, p. 11-15. Sur ces 200 adhérents, 101 résidaient à Paris ou dans la banlieue parisienne, 77 en province, deux en Algérie, un en Tunisie et un à la Réunion; la société comptait 18 membres étrangers.

51. Pour l'histoire de la Société, voir Rémy GossEz, «Éléments pour un historique de la Société d'histoire de la Révolution de 1848 et des révolutions du XIXe siècle. 1904-1940 », 1848. Révolutions et mutations au XIX siècle, 1985, p. 17-29 et Gilles CANDAR, "La "Société de 48" a cent ans ", Revue d'histoire du XIX siècle, 2005/31, p. 15-27.

52. BSHM, mai 1903, p. 94-95.

53. Ibid., p. 95.

54. Pierre CARON présenta des sources relatives à 1848, conservées aux Archives nationales, $L a$ Révolution de 1848 [R. 1848], t. I, 1904-1905, p. 57-60.

55. Voir la notice biographique sur Henry Michel par Henry SALOMON, R. 1848, t. 2, 1905-1906, p. 14-28. Voir aussi Serge AUdiER, «Éléments biographiques sur Henry Michel (1857-1904) », Henry Michel : l'individu et l'État, mis en œuvre par Serge AUDIER, Corpus, n 48, 2005, p. 13-17 et Jacqueline LALOUETTE, « Henry Michel, philosophe-historien », ibid., p. 39-60.

56. Henry MICHEL, Edgar Quinet, conférence faite à l'Université populaire de Lyon, Lyon, Stock, 1904.

57. Henry MiCHEL, « Note sur la Constitution de 1848 d'après les procès-verbaux inédits des travaux du Comité de la Constitution », R. 1848, t. I, p. 41-56; cette étude fut par ailleurs publiée en opuscule, à Lyon, par l'imprimerie Schneider, en 1904. L'ouvrage qu'Henry MicheL consacra à la loi Falloux ne fut publié qu'après sa mort, La loi Falloux, 4 janvier 1849-15 mars 1850, Paris, Hachette,1906.

58. Henry Michel, « Au fil de l'histoire », Propos de morale, Paris, Hachette, 1904, troisième volume, p. 10-11.

59. Ibid., p. 12.

60. Georges RENARD, « Comment s'est formée notre société », R. 1848, t. I, p. 1.

61. R. 1848, t. I, p. 2 .

62. Georges RENARD, « Coup d' œil sur nos travaux futurs », R. 1848, t. I, p. 11.

63. R. 1848, t. I, p. 5-9. La liste des adhérents de 1904 est reproduite à la suite de l'article de Rémy GossEZ cité note 45 .

64. Mmes Bert, Charras, Floquet, Havet, Lacroix et Melles Coucherond et Salomon. D'autres sociétaires féminines vinrent compléter cette liste, en 1906 et 1907 (Mme Zablowdowski, Melle Wassermann).

65. Il s'agit vraisemblablement de l'abbé Jules Toiton, né en 1869, ordonné prêtre en 1896, domicilié à Paris de 1903 à 1914 (fgw-cgw-picardie L.C. Généalogie en Picardie \& Val-d'Oise), qui participa au congrès ecclésiastique de Bourges (1900) et fonda L'Idéal du foyer en 1903.

66. R.1848,t. 2, 1905-1906, p. 37-44.

67. Ibid.,t. 4, 1907-1908, p. 6.

68. Ibid.,t. 3, 1906-1907, p. 277.

69. BSHM, mars 1904, p. 125. 
70. R. 1848, t. 4, 1907-1908, p. 4.

71. BSHM, mars 1908, p. 25.

72. En 1905, le président de la Société d'histoire contemporaine exprima certaines réserves relatives à la fondation de la SHR de 1848 : «C'est seulement de nos jours que la formation d'une société destinée à l'étude particulière des événements de 1848, et l'annonce de travaux isolés qu'on dit approfondis, peuvent nous faire espérer que cette partie de notre histoire nationale va enfin sortir de la période du recueillement et de l'attente. Le recul est-il réellement suffisant? On pourrait en douter; car si une nouvelle génération a pu se former avec ses appréciations personnelles, par contre, l'épreuve actuelle d'une troisième république pourra, selon les cas, rendre trop indulgent ou trop sévère pour la sœur aînée dont elle a recueilli l'héritage ", Société d'histoire contemporaine, Séance du 15 juin 1905, Discours du président, Besançon, imprimerie Jacquin, p. 2-3.

73. Toutefois, la SHR de 1848 avait un recrutement manifestement plus diversifié, puisque, outre les deux ecclésiastiques déjà mentionnés, s'y trouvaient le très catholique Georges Goyau et le protestant Franck Puaux, directeur de la Revue chrétienne.

\section{RÉSUMÉS}

De nombreuses sociétés historiques furent fondées durant le XIXe siècle, comme la Société d'histoire de France, en 1833. Ce mouvement de création s'accéléra dans le quart de siècle précédant la création de la Société des études robespierristes. Apparurent ainsi la Société de l'histoire de la Révolution française (1888), la Société d'histoire moderne (1901), la Société d'histoire de la Révolution de 1848 (1904). Ces trois sociétés donnaient la priorité à la recherche et à la publication de sources ainsi qu'à la mise en œuvre de méthodes critiques et rationnelles ; elles tenaient à développer les études locales et à permettre aux historiens provinciaux de travailler dans les meilleures conditions. Des liens forts unissaient les trois sociétés; les triples appartenances étaient d'ailleurs nombreuses, Alphonse Aulard en étant l'exemple le plus caractéristique.

Numerous historical societies were founded during the nineteenth century like the Société d'histoire de France in 1833. Their number further increased during the quarter century preceding the creation of the Société des études robespierristes. Thus emerged the Sociéte de l'histoire de la Révolution française (1888), the Société d'histoire moderne (1901), the Société d'histoire de la Révolution de 1848 (1904). These three organizations favored research, and the publication of sources as well as the application of critical and rational methods. They labored to develop local studies, and to permit local historians to work under better conditions. Strong ties united these three organizations; indeed, triple membership was frequent, Alphonse Aulard being the most characteristic example.

\section{INDEX}

Mots-clés : archives, histoire contemporaine, histoire moderne, méthodes, province, sociétés savantes, sources 
AUTEUR

\section{JACQUELINE LALOUETTE}

Université de Lille 3 - Charles de Gaulle Institut Universitaire de France 3, rue des Hautes Formes 75013 Paris Jacqueline.lalouette@wanadoo.fr 